\title{
ALGEBRAIC REPRESENTATIONS OF TEICHMÜLLER SPACE
}

\author{
By KYoJI SaIto
}

This is a summary of the paper [S5] with the same title. It is divided in two parts. In part I, we summarize some general results on the character variety of representations of a finitely generated group in $\mathrm{SL}_{2}$ ([S4]). In part II, we apply these results to the representations of surface groups in $\mathrm{SL}_{2}(\mathbf{R})$ to obtain a description of the coordinate ring for Teichmüller space as a semialgebraic set defined over $\mathbf{Z}$. Except for some minor changes, the introduction and $\S 6$ are the same as that of [ibd].

For integers $g \geq 0$ and $n \geq 0$, consider $(X, \underline{x})$, where $X$ is a compact Riemann surface of genus $g$ and $(\underline{x})=\left(x_{1}, \ldots, x_{n}\right)$ is an $n$-tuple of distinct ordered points of $X$. Recall ([Mu2]) that the moduli space $\mathfrak{M}_{g, n}$ is the set of isomorphic classes of $(X, \underline{x})$, and that the Teichmüller space $\mathcal{T}_{g, n}$ is the set of isomorphic classes of $(X, \alpha, \underline{x})$, where $\alpha$ is an isomorphism from the abstract group $\Gamma_{g, n}$ with a fixed generator system (6.2.1) to $\pi_{1}(X \backslash\{\underline{x}\})$ satisfying certain marking properties (recalled in $\left.\S 6\right)$.

We recall some well known facts about the spaces $\mathfrak{M}_{g, n}$ and $\mathcal{T}_{g, n}$, which led the author to the present work. $\mathfrak{M}_{g, n}$ has the structure of the $\mathbf{C}$-rational point set of a quasiprojective algebraic variety defined over $\mathbf{Q}([\mathrm{Mu}])$ and $\mathcal{T}_{g, n}$ has the structure of a smooth real manifold so that the natural projection $\mathcal{T}_{g, n} \rightarrow \mathfrak{M}_{g, n}$ is a ramified covering map, having $\mathrm{Out}^{+}\left(\Gamma_{g, n}\right)$ (6.2.3) as the covering transformation group. $\mathcal{T}_{g, n}$ is homeomorphic to a cell, and hence it is the universal covering space of $\mathfrak{M}_{g, n}$ in the sense of orbifold. The covering map cannot be algebraic (in any sense) when the group $\operatorname{Out}^{+}\left(\Gamma_{g, n}\right)$ is infinite, in particular when $e(X \backslash\{\underline{x}\})=2-2 g-n<0$. Therefore, it is interesting to ask for a construction of an automorphic function (or an automorphic form in a suitable sense) on $\mathcal{T}_{g, n}$ with respect to the action of $\mathrm{Out}^{+}\left(\Gamma_{g, n}\right)$. After such an attempt in [S2], the author was led to fix certain real algebraic coordinates of $\mathcal{T}_{g, n}$ which fits with that construction. This motivated the present paper.

In fact, we will realize $\mathcal{T}_{g, n}$ for $2-2 g-n<0$ as the $\mathbf{R}$-rational point set of an affine semialgebraic set defined over $\mathbf{Z}\left((6.5)\right.$ Theorem), where the coordinate ring $R_{g, n}$ is introduced abstractly as a quotient ring of the unvversal character ring $R\left(\Gamma_{g, n}, \mathrm{SL}_{2}\right)$ (introduced in part I) modulo the ideal corresponding to the parabolicity conditions on the $\mathbf{n}$ generators of $\Gamma_{g, n}$ (6.5.1). The image of the ring $R_{g, n}$ in $\mathbf{R}$ at a point $(X, \alpha, \underline{x})$ of $\mathcal{T}_{g, n} \subset \operatorname{Hom}\left(R_{g, n}, \mathbf{R}\right)$ is an invariant of the n-pointed Riemann surface $(X, \underline{x})$, which we call the ring of uniformization ((6.3.1)).

Historically, the real algebraic description of Teichmüller space was initiated by Fricke $[\mathrm{F}-\mathrm{K}]$, who used characters of discrete subgroups in $\mathrm{SL}_{2}(\mathbf{R})$ in order to determine the moduli space of compact Riemann surfaces. So some authors call it the Fricke moduli. 
Since then there have been numerous works in this direction. They lead to a semialgebraic description of Teichmüller space and to its application to the Thurston compactification $\overline{\mathcal{T}}_{g}([\mathrm{~K} 1-4],[\mathrm{H} 1],[\mathrm{Br}],[\mathrm{C}-\mathrm{S}],[\mathrm{O}],[\mathrm{S}-\mathrm{S}],[\mathrm{Ko}])$.

The present paper follows the same ideas (in particular, those of [H1]) with refinements motivated by the reasons explained above, which have lead part I ( $\$ 1-5)$ of the present paper to define the integral model of the character variety. The first 4 sections \$1-4 develop a general functorial scheme for the character variety of representations of any group $\Gamma$ in $\mathrm{SL}_{2} . \S 5$ contains a study of the character variety in case of the real field $\mathbf{R}$ as coefficients. In $\S 6$, the previous results are applied to describe the coordinate system of Teichmüller space ((6.5) Theorem). We pose two questions in $\S 6$, one on the algebraic points of $\mathcal{T}_{g, n}$ and the other on the "arithmetic compactification" of $\mathcal{T}_{g, n}$.

For those in a hurry, we suggest that they start with $\S 6$ and return to earlier sections ( $\$ 4$ and 5) when necessary.

The author was partially supported by a Grant under the Monbusho International Scientific Research Program: 04044081. The author expresses his gratitude to Prof. Mutsuo Oka for his patient help during the preparation of this paper.

\author{
Part I \\ $\S 1$ Universal representation of a group $\Gamma$ in $\mathrm{SL}_{n}$ \\ $\S 2 \mathrm{PGL}_{2}$-invariants for pairs of $2 \times 2$ matrices \\ $\S 3$ Universal character ring $R\left(\Gamma, \mathrm{SL}_{2}\right)$ \\ $\S 4$ Invariant morphism $\pi_{\Gamma}$ \\ $\S 5$ Representation variety with real coefficients \\ Part II \\ $\S 6$ Teichmüller space $\mathcal{T}_{g, n}$
}

\title{
Part I
}

Let $\Gamma$ a finitely generated group. In part $I$, we introduce the character variety $C h\left(\Gamma, \mathrm{SL}_{2}\right)$ in order to parametrize conjugacy classes of representations of $\Gamma$ in $\mathrm{SL}_{2}$ in a functorial way. Then, we specialize scalars to the real field $\mathbf{R}$ to obtain results with respect to the classical topology. Let us explain this briefly.

Let $\operatorname{Hom}\left(\Gamma, \mathrm{SL}_{n}\right)$ be the functor $R \in\{$ commutative rings with 1$\} \mapsto \operatorname{Hom}\left(\Gamma, \mathrm{SL}_{n}(R)\right)$ $\in\{$ sets $\}$. The functor is representable (see (1.3) lemma) and so, by abuse of notation, we also denote by $\operatorname{Hom}\left(\Gamma, \mathrm{SL}_{n}\right)$ the scheme over $\mathbf{Z}$ representing the functor. The group scheme $\mathrm{PGL}_{n}$ acts on $\operatorname{Hom}\left(\Gamma, \mathrm{SL}_{n}\right)$ by the adjoint action. Whether the universal categorical quotient $\operatorname{Hom}\left(\Gamma, \mathrm{SL}_{n}\right) / / \mathrm{PGL}_{n}$ (Mumford [Mu]) defined over $\mathbf{Z}$ exists or not seems to be a unsolved hard question. Instead of asking for the quotient space, we introduce the character variety $\mathrm{Ch}\left(\Gamma, \mathrm{SL}_{2}\right)$ and its discriminant subvariety $D_{\Gamma}$ abstractly as schemes over $\mathbf{Z}$ and the invariant morphism $\pi_{\Gamma}: \operatorname{Hom}\left(\Gamma, \mathrm{SL}_{2}\right) \rightarrow \mathrm{Ch}\left(\Gamma, \mathrm{SL}_{2}\right)$, for which we show that the restriction of $\pi_{\Gamma}$ on the complement of $\pi_{\Gamma}^{-1}\left(D_{\Gamma}\right)$ is a principal PGL $\mathrm{PG}_{2}$-bundle over the base space $\mathrm{Ch}^{*}\left(\Gamma, \mathrm{SL}_{2}\right):=\mathrm{Ch}\left(\Gamma, \mathrm{SL}_{2}\right) \backslash D_{\Gamma}$ with respect to the étale topology, where the inverse ımage $\pi_{\Gamma}^{-1}\left(D_{\Gamma}\right)$ is a subfunctor of $\operatorname{Hom}\left(\Gamma, \mathrm{SL}_{2}\right)$ consistıng of abel or reducible representations. We study the functor $\operatorname{Hom}^{*}\left(\Gamma, \mathrm{SL}_{2}(R)\right):=\operatorname{Hom}\left(\Gamma, \mathrm{SL}_{2}\right) \backslash$ $\pi_{\Gamma}^{-1}\left(D_{\Gamma}\right)$ consisting of universally irreducible representations in detail when the scalars are the real field $\mathbf{R}$. It is shown that the real character variety $\mathrm{Ch}^{*}\left(\Gamma, \mathrm{SL}_{2}\right)(\mathbf{R})$ de- 
composes into a disjoint union of open semi algebraic sets $H_{\Gamma}$ and $T_{\Gamma}$ so that the $\operatorname{Hom}^{*}\left(\Gamma, \mathrm{SL}_{2}(R)\right)$ and $\operatorname{Hom}^{*}(\Gamma, \mathrm{SU}(2))$ are principal $\mathrm{PGL}_{2}(\mathbf{R})$ and $U(2) /(1)$ bundles over $H_{\Gamma}$ and $T_{\Gamma}$, respectively.

$\S 1$ prepares notation concerning therepresentation variety $\operatorname{Hom}\left(\Gamma, \mathrm{SL}_{2}\right)$ over $\mathbf{Z}$. 2 studies the $\mathrm{PGL}_{2}$-invariants of $M_{2} \times M_{2}$ as building blocks. The universal character ring $R\left(\Gamma, \mathrm{SL}_{2}\right)$ is introduced in $\S 3$ abstractly so that $\mathrm{Ch}\left(\Gamma, \mathrm{SL}_{2}\right):=\operatorname{Spec}\left(R\left(\Gamma, \mathrm{SL}_{2}\right)\right)$. The principal $\mathrm{PGL}_{2}$-bundle structure on $\operatorname{Hom}^{*}\left(\Gamma, \mathrm{SL}_{2}\right)$ over $\mathrm{Ch}^{*}\left(\Gamma \cdot \mathrm{SL}_{2}\right)$ with respect to the étale topology is formulated in $\S 4$ Theorem $\mathrm{A}$, and that with respect to the classical topology is formulated in $§ 5$ Theorem C.

\section{$\S 1$. Universal representation of a group $\Gamma$ in $\mathrm{SL}_{n}$.}

This $\S$ is devoted to the preparation of notation and terminology for representation varieties. One is referred to $[\mathrm{Pr}][\mathrm{Ba}][\mathrm{L}-\mathrm{M}]$ etc.

(1.1) Let $\Gamma$ a group. As in the introduction, for a fixed $n \in \mathbf{Z}_{>\mathbf{0}}, \operatorname{Hom}\left(\Gamma, \mathrm{SL}_{n}\right)$ is the functor: $R \in\{$ commutative rings with 1$\} \mapsto \operatorname{Hom}\left(\Gamma, \mathrm{SL}_{n}(R)\right) \in\{$ sets $\}$. To fix notation for the present paper, we state precisely the representability of the functor in the next lemma.

LEMMA (the representability of $\operatorname{Hom}\left(\Gamma, \mathrm{SL}_{n}\right)$ ) 1. For a given $\Gamma$ and $n \geq 1$, there exısts a pair $\left(A\left(\Gamma, \mathrm{SL}_{n}\right), \sigma\right)$ consistıng of a commutative ring $A\left(\Gamma, \mathrm{SL}_{n}\right)$ with 1 and a representation $\sigma: \Gamma \rightarrow \mathrm{SL}_{n}\left(A\left(\Gamma, \mathrm{SL}_{n}\right)\right)$ such that for any commutative ring $R$ with 1 , the correspondence:

$$
\varphi \in \mathrm{Hom}^{r i n g}\left(A\left(\Gamma, \mathrm{SL}_{n}\right), R\right) \mapsto \varphi \circ \sigma \in \mathrm{Hom}^{g r}\left(\Gamma, \mathrm{SL}_{n}(R)\right)
$$

is a bijection.

2. The pair $\left(A\left(\Gamma, \mathrm{SL}_{n}\right), \sigma\right)$ is unique up to an isomorphism of the ring $A\left(\Gamma, \mathrm{SL}_{n}\right)$ commuting with the universal representation $\sigma$.

3. If $\Gamma$ is a finitely generated group, then $A\left(\Gamma, \mathrm{SL}_{n}\right)$ is fintely generated over $\mathrm{Z}$ and hence is noetherian.

The lemma is proven by routine arguments. Here we give an explicit description of $\left(A\left(\Gamma, \mathrm{SL}_{2}\right), \sigma\right)$. For each $\gamma \in \Gamma$, consider a $n \times n$ matrix:

$$
\sigma(\gamma):=\left(a_{i j}(\gamma)\right)_{i, j=1, \ldots, n} .
$$

Then $A\left(\Gamma, \mathrm{SL}_{n}\right)$, called the universal representation algebra, is generated by the entries $a_{i j}(\gamma), i, j=1, \ldots, n, \gamma \in \Gamma$ modulo the ideal generated by all entries of the matrices $\sigma(e)-I_{n}\left(I_{n}=\right.$ the $n \times n$ unit matrix $)$ and $\sigma(\gamma \delta)-\sigma(\gamma) \sigma(\delta)$, and by $\operatorname{det}(\sigma(\gamma))-1, \gamma \in \Gamma$.

$$
A\left(\Gamma, \mathrm{SL}_{n}\right):=\mathbf{Z}\left[a_{i j}(\gamma) \text { for } \gamma \in \Gamma \text { and } 1 \leq i, j \leq n\right] / I,
$$

where $I:=\left(a_{i j}(e)-\delta_{i j}, a_{i j}(\gamma \delta)-\sum_{k} a_{i k}(\gamma) a_{k j}(\delta), 1-\operatorname{det}\left(a_{p q}(\gamma)\right)_{p, q=1}^{n}, 1 \leq i, j \leq n\right.$ and $\gamma, \delta \in \Gamma)$. By definition, the map $\sigma: \gamma \in \Gamma \rightarrow \sigma(\gamma) \in \mathrm{SL}_{n}\left(A\left(\Gamma, \mathrm{SL}_{n}\right)\right)$ is a representation of $\Gamma$ and is called the universal representation.

(1.2) Let $\mathrm{PGL}_{n}$ be the group scheme ([SGA III], [D-G]). Its coordinate ring $A\left(\mathrm{PGL}_{n}\right)$ is given by the subring $A_{0}\left(\mathrm{GL}_{n}\right)$ of the coordinate ring $A\left(G L_{n}\right):=\mathbf{Z}\left[x_{i j}\right.$; $1 \leq i, j \leq n]$ (here $X:=\left(x_{i \jmath}\right)_{i, j=1}^{n}$ ) of $\mathrm{GL}_{n}$ consisting of homogeneous elements of 
degree 0 . The adjoint action of $\mathrm{PGL}_{n}$ on $\operatorname{Hom}\left(\Gamma, \mathrm{SL}_{n}\right)$ is written in terms of its dual action:

$$
\mathrm{Ad}: A\left(\Gamma, \mathrm{SL}_{n}\right) \longrightarrow A\left(\Gamma, \mathrm{SL}_{n}\right) \otimes \mathbf{Z} A\left(\mathrm{PGL}_{n}\right),
$$

sending an entry of $\sigma(\gamma)$ to the same entry of $X^{-1} \sigma(\gamma) X=\frac{1}{\operatorname{det}(X)} X^{*} \sigma(\gamma) X$.

(1.3) From now on, we switch to the case $n=2$. Let us list up some relations among the $\mathrm{PGL}_{2}$-invariants $\operatorname{tr}(\sigma(\gamma))$ for $\gamma \in \Gamma$ in the ring $A\left(\Gamma, \mathrm{SL}_{2}\right)$. The first one is:

$$
\operatorname{tr}(\sigma(e))=2 \text {. }
$$

The next one follows from the Caylay-Hamilton relation: $\sigma(\gamma)^{2}+\operatorname{det}(\sigma(\gamma)) I_{2}=\operatorname{tr}(\sigma(\gamma))$. $\sigma(\gamma)$. Multiply $\sigma\left(\gamma^{-1} \delta\right)$ and take traces. So we obtain:

$$
\operatorname{tr}(\sigma(\gamma \delta))+\operatorname{tr}\left(\sigma\left(\gamma^{-1} \delta\right)\right)=\operatorname{tr}(\sigma(\gamma)) \cdot \operatorname{tr}(\sigma(\delta))
$$

for $\gamma$ and $\delta \in \Gamma$ (cf.[F-K, formulas (2), p. 338]).

\section{§2. $\mathrm{PGL}_{2}$-invariants for pairs of $2 \times 2$ matrices}

We study the invariants of the diagonal adjoint action of $\mathrm{PGL}_{2}$ on the space $M_{2} \times M_{2}$ of pair $(A, B)$ of $2 \times 2$ matrices. The morphism $\tilde{\pi}: M_{2} \times M_{2} \rightarrow \mathbf{A}^{\mathbf{5}}$ given by $(A, B) \mapsto$ $(\operatorname{tr}(A), \operatorname{tr}(B), \operatorname{tr}(A B), \operatorname{det}(A), \operatorname{det}(B))$ is shown to be the universal quotient map $((2.3)$ Lemma $A, B)$. The discriminant $\Delta$ for $\tilde{\pi}$ is introduced ((2.4) Lemma $C)$ so that the $\tilde{\pi}$ is a principal $\mathrm{PGL}_{2}$-bundle on the complement of the discriminant loci $\Delta=0$ with respect to the étal topology ((2.5) Lemma D).

(2.1) Let $M_{2} \times M_{2}$ be the space of pairs of $2 \times 2$ matrices. The $X \in \mathrm{PGL}_{2}$ acts on $(A, B) \in M_{2} \times M_{2}$ from the right diagonally by letting $(A, B) \cdot \operatorname{Ad}(X):=\left(X^{-1} A X\right.$, $\left.X^{-1} B X\right)$. So we have the dual action on the coordinate ring:

$$
\operatorname{Ad}: \mathbf{Z}\left[M_{2} \times M_{2}\right] \longrightarrow \mathbf{Z}\left[M_{2} \times M_{2}\right] \otimes \mathbf{Z} A\left(\mathrm{PGL}_{2}\right)
$$

sending an entry of $(A, B)$ to the corresponding entry of $(A, B) \cdot \operatorname{Ad}(X)$ where $\mathbf{Z}\left[M_{2} \times M_{2}\right]$ is the polynomial ring generate by entries of $(A, B)$.

(2.2) Let us consider the morphism

$$
\tilde{\pi}: M_{2} \times M_{2} \longrightarrow \mathbf{A}^{5}:=\operatorname{Spec}(\mathbf{Z}[\underline{T}, \underline{D}]),
$$

where $\mathbf{Z}[\underline{T}, \underline{D}]$ denotes the polynomial ring $\mathbf{Z}\left[T_{1}, T_{2}, T_{3}, D_{1}, D_{2}\right]$ of five indeterminates and $\tilde{\pi}$ is associated to the ring homomorphism:

$$
\iota: \mathbf{Z}[\underline{T}, \underline{D}] \longrightarrow \mathbf{Z}\left[M_{2} \times M_{2}\right]
$$

given by $\iota\left(T_{1}\right):=\operatorname{tr}(A), \iota\left(T_{2}\right):=\operatorname{tr}(B), \iota\left(T_{3}\right):=\operatorname{tr}(A B), \iota\left(D_{1}\right):=\operatorname{det}(A)$ and $\iota\left(D_{2}\right):=$ $\operatorname{det}(B)$. The morphism $\tilde{\pi}$ is G-equivariant and $\mathrm{PGL}_{2}$-invariant, since $\iota$ is homogeneous w.r.t the weights $\operatorname{deg}\left(T_{1}\right)=\operatorname{deg}\left(T_{2}\right)=1$ and $\operatorname{deg}\left(T_{3}\right)=\operatorname{deg}\left(D_{1}\right)=\operatorname{deg}\left(D_{2}\right)=2$, and the Image $(\iota)$ is fixed pointwisely by the $\mathrm{PGL}_{2}$-action. By a use of an euclidean division algorithm, it is easy to prove the next lemma.

Lemma A. $\mathbf{Z}\left[M_{2} \times M_{2}\right]$ is a free module over $\mathbf{Z}[\underline{T}, \underline{D}]$.

As a consequence of Lemma $\mathbf{A}, \mathbf{Z}\left[M_{2} \times M_{2}\right]$ is farthfully flat over $\mathbf{Z}[\underline{T}, \underline{D}]$. In particular, the map $\iota(2.2 .2)$ is injective, and we regard $\mathbf{Z}[\underline{T}, \underline{D}]$ as a subring of $\mathbf{Z}\left[M_{2} \times M_{2}\right]$. 
More strongly, the next lemma says that it is the universal invariant subring with respect to the PGL-action.

LEMMA B. Let $R$ be any $\mathbf{Z}[\underline{T}, \underline{D}]$-algebra with 1 . Then

$$
R=\left(R \otimes \mathbf{Z}[\underline{T}, \underline{D}] \mathbf{Z}\left[M_{2} \times M_{2}\right]\right)^{\mathrm{PGL}_{2}} .
$$

Here, the $\mathrm{PGL}_{2}$ action on $\mathrm{Z}\left[M_{2} \times M_{2}\right]$ is extended to the tensor product by letting $\mathrm{PGL}_{2}$ act trivially on $R$.

Remark 1. It is classically well known that $\mathbf{Q}\left[M_{2} \times M_{2}\right]^{\mathrm{PGL}_{2}}$ is generated by traces $\operatorname{tr}(W)$ for $W \in\{$ the monoid generated by the $A$ and $B\}$ ([G-Y],[Pr],[W]). This is not true for the $\mathbf{Z}$-coefficient invariants. For instance, the relation: $2 \operatorname{det}(A)=\operatorname{tr}(A)^{2}-\operatorname{tr}\left(A^{2}\right)$ implies the algebraic dependence of $\operatorname{tr}\left(A^{2}\right)$ and $\operatorname{tr}(A)$ in case of char. $=2$, whereas $\operatorname{det}(A)$ and $\operatorname{tr}(A)$ are universally algebraically independent as shown in the above lemma $\mathbf{B}$.

2. Donkin [D] has shown that $\mathbf{Z}\left[M_{n} \times \cdots \times M_{n}\right]^{P G L_{n}}$ is generated by $\operatorname{tr}\left(\wedge^{2} W\right)$ for $i=1, \ldots, n$ and $W \in\left\{\right.$ the monoid generated by $\left.A_{1}, \ldots, A_{m}\right\}$, where we denote by $M_{n} \times \cdots \times M_{n}$ the space of m-tuple $n \times n$ matrices $\left(A_{1}, \ldots, A_{m}\right)$.

(2.4) We introduce the discriminant $\Delta=\Delta(A, B)$ of the morphism $\tilde{\pi}$. For a $2 \times 2$ matrix $A$, let $A^{*}$ denote its adjoint matrix so that $A^{*} A=A A^{*}=\operatorname{det}(A) I_{2}$ and $A+A^{*}=$ $\operatorname{tr}(A) I_{2}$.

Definition. The discrimınant for $\tilde{\pi}$ is the polynomial:

$$
\begin{aligned}
\Delta(A, B): & =\operatorname{tr}\left(A B A^{*} B^{*}\right)-\operatorname{tr}\left(A A^{*} B B^{*}\right) \\
& =T_{1}^{2} D_{2}+T_{2}^{2} D_{1}+T_{3}^{2}-T_{1} T_{2} T_{3}-4 D_{1} D_{2} .
\end{aligned}
$$

A justification for this name is in the next lemma C. Let $J$ be the ideal of $\mathbf{Z}\left[M_{2} \times M_{2}\right]$ generated by all $5 \times 5$ minors of the Jacobian matrix of the morphism $\widetilde{\pi}(2.2 .1)$ :

$$
\frac{\partial\left(T_{1}, T_{2}, T_{3}, D_{1}, D_{2}\right)}{\partial(a, b, c, d, e, f, g, h)}=\left(\begin{array}{cccccccc}
1 & 0 & 0 & 1 & 0 & 0 & 0 & 0 \\
0 & 0 & 0 & 0 & 1 & 0 & 0 & 1 \\
e & g & f & h & a & c & b & d \\
d & -c & -b & a & 0 & 0 & 0 & 0 \\
0 & 0 & 0 & 0 & h & -g & -f & e
\end{array}\right),
$$

where $a, \cdots, h$ are the entries of $(A, B)$.

LEMMA C. The intersection of the Jacobi ideal $J$ with the invariant subring $\mathbf{Z}[\underline{T}, \underline{D}]$ is a principal ideal generated by the discriminant.

$$
J \cap \mathbf{Z}[\underline{T}, \underline{D}]=(\Delta) .
$$

(2.5) Let us denote by $D_{\Delta}$ the divisor of $\mathbf{A}^{5}=\operatorname{Spec}(\mathbf{Z}[\underline{T}, \underline{D}])$ defined by the equation $\Delta=0$. Owing to the (2.4.2), the Jacobian criterion implies that the morphism $(2.2 .1)$ on the complement of $\widetilde{\pi}^{-1}\left(D_{\Delta}\right)$

$$
\tilde{\pi}: M_{2} \times M_{2} \backslash \tilde{\pi}^{-1}\left(D_{\Delta}\right) \rightarrow \mathbf{A}^{5} \backslash D_{\Delta}
$$


is smooth. More strongly, we show in the next lemma that $\tilde{\pi}$ is a principal $\mathrm{PGL}_{2}$-bundle, to which the proof of main theorem $\mathrm{A}$ in $\S 4$ is reduced.

LEMMA D. The morphism (2.5.1) is a principal $\mathrm{PGL}_{2}$-bundle with respect to the étal topology.

\section{§3. The universal character ring $R\left(\Gamma, \mathrm{SL}_{2}\right)$.}

We introduce the universal character ring $R\left(\Gamma, \mathrm{SL}_{2}\right)$ by generators and relations in terms of $\Gamma$. The goal is the formula (3.4.3), which is used in the proof of the main theorem $\mathrm{B}$ in $\S 4$.

(3.1) Definition. The universal character ring $R\left(\Gamma, \mathrm{SL}_{2}\right)$ of representations of $\Gamma$ in $\mathrm{SL}_{2}$ is a ring generated by the indeterminates $s(\gamma)$ for $\gamma \in \Gamma$ and divided by the ideal generated by $s(e)-2(e=$ the unit of $\Gamma)$ and by $s(\gamma) s(\delta)-s(\gamma \delta)-s\left(\gamma^{-1} \delta\right)$ for all $\gamma, \delta \in \Gamma$.

$$
R\left(\Gamma, \mathrm{SL}_{2}\right):=\mathbf{Z}[s(\gamma), \gamma \in \Gamma] /\left(s(e)-2, s(\gamma) s(\delta)-s(\gamma \delta)-s\left(\gamma^{-1} \delta\right)\right)
$$

(3.2) The ring $R\left(\Gamma, \mathrm{SL}_{2}\right)$ is finitely generated over $\mathbf{Z}$ if $\Gamma$ is finitely generated as shown in the next lemma. Such finiteness was asserted for the ring of traces of $\mathrm{SL}_{2}$ by Fricke $[\mathrm{F}-\mathrm{K}]$ and proven in $[\mathrm{H},(2, \mathrm{f})],[\mathrm{Ho}, \mathrm{Theo} .3 .1$.$] and [\mathrm{C}-\mathrm{S}]$.

Proposition. Let A be a linearly ordered subset of $\Gamma$, which generates $\Gamma$. Then $R\left(\Gamma, \mathrm{SL}_{2}\right)$ is generated by $G:=\cup_{m \in \mathbf{N}}\left\{s\left(\alpha_{1} \cdots \alpha_{m}\right) \mid \alpha_{i} \in A, \alpha_{1}<\cdots<\alpha_{m}\right\}$ over $\mathbf{Z}$.

CoROLlaRY 1. If the group is finitely generated then the $R\left(\Gamma, \mathrm{SL}_{2}\right)$ is finitely generated over $\mathbf{Z}$. Hence it is noetherian.

(3.3) For $\alpha$ and $\beta \in \Gamma$, define the discriminant $\Delta(\alpha, \beta) \in R\left(\Gamma, \mathrm{SL}_{2}\right)$ by

$$
\begin{aligned}
\Delta(\alpha, \beta): & =s\left(\alpha \beta \alpha^{-1} \beta^{-1}\right)-2 \\
& =s(\alpha)^{2}+s(\beta)^{2}+s(\alpha \beta)^{2}-s(\alpha) s(\beta) s(\alpha \beta)-4 .
\end{aligned}
$$

This definition of $\Delta$ is pararell to that in $\mathbf{Z}[\underline{T}, \underline{D}]$ (2.4.1). In fact, we shall confuse them in a proof of the main theorem $B$ in $\S 4$. The discriminant polynomial has been studied extensively by $R$. Fricke $[F]$ and others.

(3.4) An importance of the discriminant is explained in the next lemma, which plays an essential role in a proof of the main theorem $B$ in $\S 4$.

Definition. Let $M$ be a $R\left(\Gamma, \mathrm{SL}_{2}\right)$-module. A map $h: \Gamma \rightarrow M$ is called a form with values in $M$, if for any $\gamma$ and $\delta \in \Gamma$ one has a relation:

$$
h(\gamma \delta)+h\left(\gamma^{-1} \delta\right)=s(\gamma) h(\delta)
$$

LEMMA. Let $h$ be a form with values in $M$. Suppose $h(e)=h(\alpha)=h(\beta)=h(\alpha \beta)=$ 0 for some $\alpha$ and $\beta \in \Gamma$. Then, for any $\gamma \in \Gamma$, one has

$$
\Delta(\alpha, \beta) h(\gamma)=0 \text {. }
$$

Therefore, the composition of $h$ with the localization $M \rightarrow M_{\Delta(\alpha, \beta)}$ is identically zero. 
Corollary. For any $\alpha, \beta, \gamma$ and $\delta \in \Gamma$, one has

$$
s(\gamma \delta)=-\frac{1}{\Delta(\alpha, \beta)}(s(\gamma), s(\gamma \alpha), s(\gamma \beta), s(\gamma \alpha \beta)) \cdot T \cdot{ }^{t}(s(\delta), s(\alpha \delta), s(\beta \delta), s(\alpha \beta \delta))
$$

in the localization $R\left(\Gamma, \mathrm{SL}_{2}\right)_{\Delta(\alpha, \beta)}$, where $T$ is a matrix in $M_{4}\left(R\left(\Gamma, \mathrm{SL}_{2}\right)\right)$ such that

$$
T \cdot\left(s\left(\xi_{i} \xi_{\jmath}\right)\right)_{i, j=1}^{4}=-\Delta(\alpha, \beta) I_{4} \quad \text { for } \quad \xi_{1}=e, \xi_{2}=\alpha, \xi_{3}=\beta \quad \text { and } \quad \xi_{4}=\alpha \beta .
$$

We shall see that the above is a key lemma showing that the system $\{s(\gamma), \gamma \in \Gamma\}$ satisfies any algebraic relations which is satisfied by the system $\{\operatorname{tr}(\sigma(\gamma)), \gamma \in \Gamma\}$ of characters so far as $\Delta(\alpha, \beta)$ is invertible for some $\alpha, \beta \in \Gamma$.

(3.5) Remark 1. The study of the algebra of traces characters of representations of a group $\Gamma$ into $\mathrm{SL}_{2}$ started by Voigt and Fricke and is developed by many authors Helling, Horowitz, Magnus, Bass, Lubotsky, Procesi, Platonov and others. See references of the quoted papers.

2. Let $F_{n}$ be a free group generated by $\mathrm{n}$ elements. Magnus called the homomorphic image of the ring $R\left(F_{n}, \mathrm{SL}_{2}\right)$ in the ring of functions on the representation space $\operatorname{Hom}\left(F_{n}, \mathrm{SL}_{2}(\mathrm{C})\right)$ the ring of Fricke characters [Ma]. We do not know whether the homomorphism has non trivial kernel or not.

\section{§4. The invariant morphism $\pi_{\Gamma}$}

We introduce the character variety $\mathrm{Ch}\left(\Gamma, \mathrm{SL}_{2}\right)$, the $\mathrm{PGL}_{2}$-invariant morphism $\pi_{\Gamma}$ $: \operatorname{Hom}\left(\Gamma, \mathrm{SL}_{2}\right) \rightarrow \mathrm{Ch}\left(\Gamma, \mathrm{SL}_{2}\right)$ and the discriminant $D_{\Gamma} \subset \mathrm{Ch}\left(\Gamma, \mathrm{SL}_{2}\right)$ in (4.1). The inverse image $\pi^{-1}\left(D_{\Delta}\right)$ consist of the representations $\rho$ such that $\rho(\Gamma)$ is either abelian or reducible ((4.2) Assertion). Then the goal result ((4.3) Theorem A) states that the restriction of $\pi_{\Gamma}$ on the complement of $\pi^{-1}\left(D_{\Delta}\right)$ is a principal $\mathrm{PGL}_{2}$-bundle with respect to the étal topology.

(4.1) Let $\Gamma$ be a group. Put

$$
\operatorname{Ch}\left(\Gamma, \mathrm{SL}_{2}\right):=\operatorname{Spec}\left(R\left(\Gamma, \mathrm{SL}_{2}\right)\right)
$$

where $R\left(\Gamma, \mathrm{SL}_{2}\right)$ is defined in (3.1). Recall the fact that the functor $\operatorname{Hom}\left(\Gamma, \mathrm{SL}_{2}\right)$ is identified with the affine scheme for the universal reperesentation ring $A\left(\Gamma, \mathrm{SL}_{2}\right)$ (cf (1.3) lemma). Then the invariant morphism is defined

$$
\pi_{\Gamma}: \operatorname{Hom}\left(\Gamma, \mathrm{SL}_{2}\right) \rightarrow \mathrm{Ch}\left(\Gamma, \mathrm{SL}_{2}\right) .
$$

through the ring homomorphism

$$
\Phi: R\left(\Gamma, \mathrm{SL}_{2}\right) \longrightarrow A\left(\Gamma, \mathrm{SL}_{2}\right), \quad s(\gamma) \longmapsto \operatorname{tr}(\sigma(\gamma))
$$

Comparing relations (3.1.1) with (1.3.1) and (1.3.2), $\Phi$ is well defined. The discrimınant subvariety $\subset \mathrm{Ch}\left(\Gamma, \mathrm{SL}_{2}\right)$ of the morphism $\pi_{\Gamma}$ is defined as the intersection of the divisors:

$$
D_{\Gamma}:=\bigcap_{\alpha, \beta \in \Gamma} D_{\Delta(\alpha, \beta)}
$$

associated to the discriminant $\Delta(\alpha, \beta) \in R\left(\Gamma, \mathrm{SL}_{2}\right)(3.3 .1)$.

(4.2) We first characterize the inverse image $\widetilde{\pi}^{-1}\left(D_{\Gamma}\right)$ of the discriminant in terms of representation of $\Gamma$. 
Assertion. Let $\mathfrak{p} \in \operatorname{Spec}\left(A\left(\Gamma, \mathrm{SL}_{2}\right)\right)$. Then $\mathfrak{p}$ belongs to $\tilde{\pi}^{-1}\left(D_{\Delta}\right)$, if and only if the image $\sigma_{\mathfrak{p}}(\Gamma)$ in $\mathrm{SL}_{2}\left(k_{\mathfrak{p}}\right)$ of the group $\Gamma$ is either abelian or reducible, where $k_{\mathfrak{p}}$ is the field of quotients of the integral domain $A\left(\Gamma, \mathrm{SL}_{2}\right) / \mathfrak{p}$ and $\sigma_{\mathfrak{p}}: \Gamma \rightarrow \mathrm{SL}_{2}\left(k_{\mathfrak{p}}\right)$ is a representation obtained by a specialization of the unvversal at $\mathfrak{p}$.

(4.3) Let us state one main result of the part I.

THEOREM A. The restriction of the invariant morphism $\pi_{\Gamma}$ to the complement of the inverse image $\widetilde{\pi}^{-1}\left(D_{\Gamma}\right)$ of the discriminant is a principal $\mathrm{PGL}_{2}$-bundle with respect to the étal topology defined over $\mathbf{Z}$.

Proof. By definition of $D_{\Gamma}$, the $\mathrm{Ch}^{*}\left(\Gamma, \mathrm{SL}_{2}\right):=\mathrm{Ch}\left(\Gamma, \mathrm{SL}_{2}\right) \backslash D_{\Gamma}$ admits anaffine open covering $\bigcup_{\alpha, \beta \in \Gamma} \operatorname{Spec}\left(R\left(\Gamma, \mathrm{SL}_{2}\right)_{\Delta(\alpha, \beta)}\right)$. Then the proof is reduced to each affine open piece. It is a consequence of the next Theorem $B$.

(4.4) For any fixed pair $\alpha$ and $\beta$ of $\Gamma$, consider the $P_{G L}$-equivariant morphism $\operatorname{Hom}\left(\Gamma, \mathrm{SL}_{2}\right) \rightarrow M_{2} \times M_{2}$ and a morphism $h_{\alpha \beta}: \operatorname{Ch}\left(\Gamma, \mathrm{SL}_{2}\right) \rightarrow \mathbf{A}^{\mathbf{5}}=\operatorname{Spec}(\mathbf{Z}[\underline{T}, \underline{D}])$ defined by the coordinate ring homomorphisms $\mathbf{Z}\left[M_{2} \times M_{2}\right] \rightarrow A\left(\Gamma, \mathrm{SL}_{2}\right)$ and $\mathbf{Z}(T, D) \rightarrow$ $R\left(\Gamma, \mathrm{SL}_{2}\right)$ given by

$$
\begin{aligned}
& A \mapsto \sigma(\alpha) \quad \text { and } \quad B \mapsto \sigma(\beta), \\
& T_{1} \mapsto s(\alpha), T_{2} \mapsto s(\beta), T_{3} \mapsto s(\alpha \beta), D_{1} \mapsto 1 \quad \text { and } \quad D_{2} \mapsto 1
\end{aligned}
$$

so that the next diagram becomes commutative.

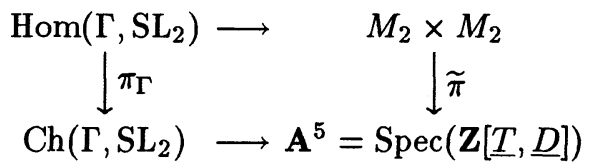

Here we remark that the discriminant $\Delta(\alpha, \beta)$ is the pull back of $\Delta(A, B)$ (2.4.1). By abuse of notation, we shall denote both of them by $\Delta$.

Theorem B. The diagram (4.4.3) is Cartesian on the complement of the discriminant loci $D_{\Delta}=\{\Delta=0\}$. That is the localization of the homomorphism

$$
\Psi_{\alpha, \beta}: R\left(\Gamma, \mathrm{SL}_{2}\right) \otimes \mathbf{Z}[\underline{T}, \underline{D}] \mathbf{Z}\left[M_{2} \times M_{2}\right] \rightarrow A\left(\Gamma, \mathrm{SL}_{2}\right)
$$

(obtaıned from (4.1.3), (4.4.1) and (4.4.2)) by $\Delta$ is an ısomorphısm.

Theorem A follows from Theorem B together with (2.5) Lemma D. The theorem $\mathrm{B}$ is proved by constructing the inverse homomorphism of the localization $\left(\Psi_{\alpha, \beta}\right)_{\Delta}$ of (4.4.4) by $\Delta$. This is equivalent to the construction of a representation $\sigma^{*}$ for a prescribed system of characters $\{s(\gamma), \gamma \in \Gamma\}$ and a pair of matrix $(A, B) \in M_{2} \times M_{2}$ over the same point of $\mathbf{A}^{\mathbf{5}} \backslash\{\Delta=0\}$ such that $\operatorname{tr}\left(\sigma^{*}(\gamma)\right)=s(\gamma)$ and $\left(\sigma^{*}(\alpha), \sigma^{*}(\beta)\right)=(A, B)$. Actually this is achieved by the formula

$$
\sigma^{*}(\gamma):=-\frac{1}{\Delta(\alpha, \beta)}\left(I_{2}, A, B, A B\right) \cdot T \cdot{ }^{t}(s(\gamma), s(\alpha \gamma), s(\beta \gamma), s(\alpha \beta \gamma))
$$

where $\mathrm{T}$ is the same $4 \times 4$ matrix in $M_{4}\left(R\left(\Gamma, \mathrm{SL}_{2}\right)\right)$ in (3.4.3). The multiplicativity $\sigma^{*}(\gamma \delta)=\sigma^{*}(\gamma) \sigma^{*}(\delta)$ is shown by a use of the formula (3.4.3). 


\section{$\S 5$. Representation variety with real coefficients}

This is the goal of the part I, where we show the principal $\mathrm{PGL}_{2}(\mathbf{R})$-bundle structure on $\mathrm{Hom}^{*}\left(\Gamma, \mathrm{SL}_{2}(\mathbf{R})\right)$ over an open semialgebraic set $H_{\Gamma}$ in the real charcter variety with respect to the classical topology (5.5) Theorem C. For the purpose we analize the discriminant $\Delta$ over the real number field $\mathbf{R}((5.3)$ Lemma E). See also $[K],[G],[H 1]$ and $[\mathrm{Ko}]$ for the geometry of the discriminant.

(5.1) Consider the invariant map $\tilde{\pi}(2.2 .1)$ over the real number field $\mathbf{R}$.

$$
\tilde{\pi}: M_{2}(\mathbf{R}) \times M_{2}(\mathbf{R}) \longrightarrow \mathbf{A}^{5}(\mathbf{R}):=\operatorname{Hom}(\mathbf{Z}[\underline{T}, \underline{D}], \mathbf{R})
$$

given by $\tilde{\pi}(A, B):=(\operatorname{tr}(A), \operatorname{tr}(B), \operatorname{tr}(A B), \operatorname{det}(A), \operatorname{det}(B)$. Consider also the real loci of the discriminant $\Delta(2.4)$.

$$
\widetilde{D}_{\Delta}(\mathbf{R}):=\left\{\phi \in \mathbf{A}^{5}(\mathbf{R}):=\Delta(\phi):=\phi(\Delta)=0\right\} .
$$

(5.2) Let us introduce an open semialgebraic subset of $\mathbf{A}^{5}(\mathbf{R})$ :

$$
\widetilde{T}_{\Delta}:=\left\{\delta_{1}<0, \delta_{2}<0, \delta_{3}<0, \Delta<0\right\}
$$

where

$$
\delta_{1}=T_{1}^{2}-4 D_{1}, \quad \delta_{2}=T_{2}^{2}-4 D_{2} \quad \text { and } \quad \delta_{3}=T_{3}^{3}-4 D_{1} D_{2} .
$$

are the discriminants for the characteristic polynomials of $\mathrm{A}, \mathrm{B}$ and $\mathrm{AB}$, respectively. The following facts are shown by direct elementary calculations

LEMMA E. i) $\widetilde{T}_{\Delta}$ is a convex connected component of $\mathbf{A}^{\mathbf{5}}(\mathbf{R}) \backslash \widetilde{D}_{\Delta}$.

ii) The image of $\widetilde{\pi}(5.1 .1)$ is equal to the complement of $\widetilde{T}_{\Delta}$. That is: $\mathbf{A}^{5}(\mathbf{R}) \backslash \widetilde{T}_{\Delta}=$ $\tilde{\pi}\left(M_{2}(\mathbf{R}) \times M_{2}(\mathbf{R})\right)$.

Let us decompose the space $\mathbf{A}^{5}(\mathbf{R})$ into semialgebraic sets:

$$
\mathbf{A}^{5}(\mathbf{R})=\tilde{D}_{\Delta} \amalg \tilde{H}_{\Delta} \amalg \tilde{T}_{\Delta},
$$

where $\widetilde{H}_{\Delta}:=\mathbf{A}^{5}(\mathbf{R}) \backslash\left(\widetilde{D}_{\Delta} \amalg \widetilde{T}_{\Delta}\right)$ is a union of connected components of $\mathbf{A}^{5}(\mathbf{R}) \backslash \widetilde{D}_{\Delta}$. Then the lemma $\mathrm{E}$ ii) is paraphrase as: $\operatorname{Image}(\widetilde{\pi})=\widetilde{D} \amalg \widetilde{H}_{\Delta}$.

(5.3) For any given $\alpha, \beta \in \Gamma$, consider the homomorphism $F_{2} \rightarrow \Gamma$ associating the two generators of $F_{2}$ to $\alpha$ and $\beta$. This induces the diagram (4.4.3)

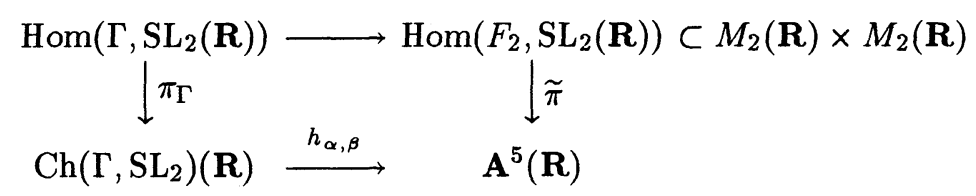

where the morphism $h_{\alpha, \beta}$ is defined by (4.4.2) and $\mathrm{Ch}\left(\Gamma, \mathrm{SL}_{2}\right)(\mathbf{R})=\operatorname{Hom}\left(R\left(\Gamma, \mathrm{SL}_{2}\right), \mathbf{R}\right)$ is the real character variety. The fact that (5.3.1) is Cartesian outside of the loci $\Delta=0$ ((4.4) Theorem B) together with Lemma E implies the following disjoint decomposition:

$$
\mathrm{Ch}\left(\Gamma\left(\Gamma, \mathrm{SL}_{2}\right)(\mathbf{R})=D_{\Gamma}(\mathbf{R}) \amalg H_{\Gamma} \amalg T_{\Gamma},\right.
$$

where

$$
\begin{aligned}
& D_{\Gamma}:=\left\{t \in \operatorname{Hom}\left(R\left(\Gamma, \mathrm{SL}_{2}\right), \mathbf{R}\right) \mid h_{\alpha, \beta}(t) \in D_{\Gamma}(\mathbf{R}) \text { for } \forall \alpha, \beta \in \Gamma\right\} \\
& H_{\Gamma}:=\left\{t \in \operatorname{Hom}\left(R\left(\Gamma, \mathrm{SL}_{2}\right), \mathbf{R}\right) \mid \exists \alpha, \beta \in \Gamma \text { such that } h_{\alpha, \beta}(t) \in \widetilde{H}_{\Delta}\right\} \\
& \left.T_{\Gamma}:=t \in \operatorname{Hom}\left(R\left(\Gamma, \mathrm{SL}_{2}\right), \mathbf{R}\right) \mid \exists \alpha, \beta \in \Gamma \quad \text { such that } h_{\alpha, \beta}(t) \in \widetilde{T}_{\Delta}\right\}
\end{aligned}
$$


By definition $D_{\Gamma}(\mathbf{R})$ is Zariski closed, and $H_{\Gamma}$ and $T_{\Gamma}$ are open semialgebraic sets. In fact, due to the basis theorem of Hilbert, one can find a finite system $\left\{\left(\alpha_{i}, \beta_{i}\right)\right\}_{i \in I}$ such that $D_{\Gamma}(\mathbf{R})=\bigcap_{i \in I}\left\{\Delta\left(\alpha_{i}, \beta_{i}\right)=0\right\}$. Then for the same index set I, one can show the equalities: $H_{\Gamma}=\bigcap_{i \in I} h_{\alpha_{i}, \beta_{i}}{ }^{-1}\left(\widetilde{H}_{\Delta}\right), T_{\Gamma}=\cup_{i \in I} h_{\alpha_{i}, \beta_{i}}{ }^{-1}\left(\widetilde{T}_{\Delta}\right)$.

(5.4) Let us determine the image set of $\pi_{\Gamma}(\mathbf{R})$ as a semialgebraic set up to the discriminant in the next lemma. It is shown by the fact that (5.3.1) is Cartesian ((4.4) Theorem B) together with the lemma $\mathrm{E}$.

LEMMA F. Let $\pi_{\Gamma}(\mathbf{R})$ be the real invariant morphism (4.1.2). Then

$$
\operatorname{Image}\left(\pi_{\Gamma}(\mathbf{R})\right) \backslash D_{\Gamma}(\mathbf{R})=H_{\Gamma} \text {. }
$$

A meaning of the set $T_{\Gamma}$ is given by the next lemma. Since $\mathrm{SU}(2) \subset \mathrm{SL}_{2}(\mathbf{C})$, the restriction of $\pi_{\Gamma}(\mathbf{C})$ induces the following map:

$$
\begin{array}{cc}
u_{\Gamma}: \operatorname{Hom}(\Gamma, \mathrm{SU}(2)) & \longrightarrow \mathrm{Ch}\left(\Gamma, \mathrm{SL}_{2}\right)(\mathbf{R}) \\
\rho & \mapsto(s(\gamma) \mapsto \operatorname{tr}(\rho(\gamma))
\end{array}
$$

LEMмA G. The image set of the morphism $u_{\Gamma}$ is given by

$$
\operatorname{Image}\left(u_{\Gamma}\right) \backslash D_{\Gamma}(\mathbf{R})=T_{\Gamma} \text {. }
$$

(5.5) This is the goal of the part I. Combining the above (5.4.1) and (5.4.3) with the $\S 4$ Theorem A, we obtain principal bundles with respect to the classical topology.

THEOREM C. The restrictions of the maps $\pi_{\Gamma}$ (4.1.2) and $u_{\Gamma}$ (5.4.2) to the set of nonabel and irreducible representations $\operatorname{Hom}\left(\Gamma, \mathrm{SL}_{2}(\mathbf{R})\right):=\operatorname{Hom}\left(\Gamma, \mathrm{SL}_{2}(\mathbf{R})\right) \backslash \pi_{\Gamma}^{-1}$ $\left(D_{\Gamma}(\mathbf{R})\right)$ and $\operatorname{Hom}^{*}(\Gamma, \mathrm{SU}(2)):=\operatorname{Hom}(\Gamma, \mathrm{SU}(2)) \backslash u_{\Gamma}^{-1}\left(D_{\Gamma}(\mathbf{R})\right)$ are a principal $\mathrm{PGL}_{2}$ (R)-bundle and a principal $U(2) / U(1)$-bundle over the open semialgebraic sets $H_{\Gamma}$ and $T_{\Gamma}(5.3 .2)$ of the real character variety $\mathrm{Ch}\left(\Gamma, \mathrm{SL}_{2}\right)(\mathbf{R})$, respectively.

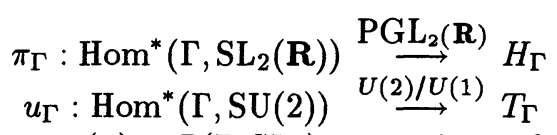

The value of a coordinate $s(\gamma) \in R\left(\Gamma, \mathrm{SL}_{2}\right)$ at a point $t$ of the base space is equal to the character $\operatorname{tr}(\rho(\gamma))$ for any representation $\rho$ in its fiber $\pi_{\Gamma}^{-1}(t)\left(\right.$ resp. $\left.u_{\Gamma}^{-1}(t)\right)$.

\section{Part II}

Fricke [F-K] started to describe the moduli of Riemann surfaces in terms of characters of descrete subgroups in $\mathrm{PGL}_{2}(\mathbf{R})$. The idea was followed by many authors, which lead to a semialgebraic description of the Teichmüller space (which sometimes is called the Fricke moduli space) (see [K], [H], [C-S], [O], [S-S], [Ko] and others).

Following the same idea, we describe $\mathcal{T}_{g, n}(=$ the Teichmüller space for Riemann surfaces $X$ of genus $g$ with $n$-distinct ordered set $\{\underline{x}\}$ of point of $X$ ) as a component of the R-rational point set of an affine scheme $\operatorname{Spec}\left(R_{g, n}\right)\left((6.5)\right.$ Theorem). Here $R_{g, n}$ is the quotient ring of the universal character ring $R\left(\Gamma_{g, n}, S L_{2}\right)$ divided by the ideal coming from the parbolicity condition at $n$ points (6.5.1) such that the values (in $\mathbf{R}$ ) of $R_{g, n}$ at a point $t$ of $\mathcal{T}_{g, n}$ is an invariant of $(X, \underline{x})$, called the ring of uniformization $R(x, \underline{x})$ introduced in (6.3.1). The description leads to some questions in (6.8) and (6.9). 


\section{§6. Teichmüller spaces $\mathcal{T}_{g, n}$}

(6.1) First we recall definitions of the moduli space of curves and the Teichmüller space from Mumford [Mu2, Lec.II].

For non-negative integers $g$ and $n$, we put

$$
\mathfrak{M}_{g, n}:=\left\{\begin{array}{l}
\text { isomorphism classes of }\left(X, x_{1}, \ldots, x_{n}\right) \\
\text { where } X \text { is a curve } \mathbf{C} \text { of genus } g, \text { and } x_{1}, \ldots, x_{n} \\
\text { are disctinct ordered points of } X .
\end{array}\right\}
$$

The $\mathfrak{M}_{g, n}$ is equiped with a structure of the $\mathbf{C}$-rational point set of a quasi projective algebraic variety defined over $\mathbf{Q}$ (see $[\mathrm{Mu} 1,2])$. The structure is characterized by the property that for any smooth family $\pi: X \rightarrow S$ of curves of genus $g$ with $n$ disjoint sections, the induced map $S \rightarrow \mathfrak{M}_{g, n}$, associating to each $s \in S$ the class of the fiber $\pi^{-1}(s)$, is a morphism of the variety.

(6.2) It is well known that the $\mathfrak{M}_{g, n}$ has singular points. Taking so called the level $N$ structure $(N \geq 3)$, we obtain a smooth space $\mathfrak{M}_{g, n}(N)$ which covers $\mathfrak{M}_{g, n}$ ramifying along the singularities. Among such smooth covering spaces, the Teichmuller space gives the most universal one. So let us introduce the Teichmüller space and the mapping class group acting on it. First, introduce an abstract group

$$
\Gamma_{g, n}:=\left\langle a_{1}, \ldots, a_{g}, b_{1}, \ldots, b_{g}, c_{1}, \ldots, c_{n} \mid\left(\prod_{i=1}^{g} a_{i} b_{i} a_{i}^{-1} b_{i}^{-1}\right) \prod_{j=1}^{n} c_{j}\right\rangle
$$

which is isomorphic to the fundamental group of $n$-punctured oriented surface of genus $g$. Then the Teichmüller space is introduced by

$$
\mathcal{T}_{g, n}:=\left\{\begin{array}{c}
\text { set of }\left(X, \alpha, x_{1}, \cdots, x_{n}\right), \text { where } X \text { is a curve } / \mathbf{C} \text { of genus } g, x_{1}, \cdots, x_{n} \text { are } \\
\text { distinct points of } X \text { and } \alpha: \Gamma_{g, n} \simeq \pi\left(X \backslash\left\{x_{1}, \cdots, x_{n}\right\}\right) \text { is an isomoprhism } \\
\text { such that } \alpha\left(c_{i}\right) \text { is freely homotopic to a small loop around } x_{i} \text { in positive } \\
\text { sense [and if } n=0, \alpha \text { is orientation preserving], modulo the equivalence } \\
(X, \alpha, \underline{x}) \sim\left(X^{\prime}, \alpha^{\prime}, \underline{x}^{\prime}\right) \mid \text { if there is an isomorphism } \phi: X \simeq X^{\prime} \text { such that } \\
\phi\left(x_{i}\right)=x_{\imath}^{\prime} \text { and such that } \phi_{*} \circ \alpha \text { differs from } \alpha^{\prime} \text { by an inner automorphism. }
\end{array}\right\}
$$

This set obtains complex manifold structure via deformation theory of compact complex manifold [K-S] in such manner that the natural projection $\mathcal{T}_{g, n} \rightarrow \mathfrak{M}_{g, n}$ is a ramified complex analytic covering map. This is realized as the quotient by the mapping class group action given below. Put,

$$
\operatorname{Out}^{+}\left(\Gamma_{g, n}\right):=\left\{\sigma \in \operatorname{Aut}\left(\Gamma_{g, n}\right) \mid \begin{array}{l}
\sigma\left(c_{i}\right) \text { is conjugate to } c_{i} \text { (and if } \\
n=0, \sigma \text { is orientation preserving) }
\end{array}\right\} / \text { Inner automorph. }
$$

(This is an abuse of notation, since the abstract group $\Gamma_{g, n}$ alone does not determine Out $\left.{ }^{+}\left(\Gamma_{g, n}\right)\right)$. Then $\mathrm{Out}^{+}\left(\Gamma_{g, n}\right)$ acts on $\mathcal{T}_{g, n}$ properly discontinuously by letting $\sigma \cdot(X, \alpha, \underline{x})=\left(X, \alpha \circ \sigma^{-1}, \underline{x}\right)$ such that one has:

$$
\mathfrak{M}_{g, n} \simeq \mathrm{Out}^{+}\left(\Gamma_{g, n}\right) \backslash \mathcal{T}_{g, n} .
$$

From now on, let us assume that the Euler number $2-2 g-n$ of $X \backslash\{\underline{x}\}$ is negative. 
(6.3) Since a (compact) curve/C is an equivalent data of a compact Riemann surface, we switch the terminologies from now on. Let $(X, \underline{x})$ be a pair of a compact Riemann surface $X$ of genus $g$ with $n$ distinct ordered points $\underline{x}$ on it. The uniformization theorem of Poincaré asserts that there exists a discrete subgroup $\Gamma$ of $\operatorname{PSL}_{2}(\mathbf{R})$ acting on the complex upper half plane $\mathbf{H}:=\{z \in \mathbf{C} \mid \operatorname{Im}(z)>0\}$ fixed point freely such that $X \backslash$ $\left\{x_{1}, \cdots, x_{n}\right\}$ is complex analytically isomorphics to the quotient $\Gamma \backslash \mathbf{H}$. In fact, then, $\Gamma$ is naturally isomrophic to $\pi_{1}\left(X \backslash\left\{x_{1}, \cdots, x_{n}\right\}\right) \stackrel{\alpha}{\simeq} \Gamma_{g, n}$ in such manner that the $\bar{\rho}\left(c_{i}\right) \in \Gamma$ corresponding to $c_{i}$ are parabolic (i.e. $\left|\operatorname{tr} \bar{\rho}\left(c_{i}\right)\right|=2$ ).

For the given $(X, \underline{x})$, the group $\Gamma \subset \mathrm{PSL}_{2}(\mathbf{R})$ of uniformization of $X \backslash\{\underline{x}\}$ is unique up to conjugacy by the adjoint action of $\mathrm{PGL}_{2}(\mathbf{R})$. Therefore, associated to the $(X, \underline{x})$, we introduce the following subring of $\mathbf{R}$.

$$
R(X, \underline{x}):=\text { subring of } \mathbf{R} \text { generated by } \pm \operatorname{tr}(\gamma) \text { for }{ }^{\forall} \gamma \in \Gamma \text { over } \mathbf{Z} \text {. }
$$

This ring does not depend on a choice of the group $\Gamma$ but depends only on the equivalence class of $(X, \underline{x})$, that is, only on the point in $\mathfrak{M}_{g, n}$ associated to $(X, \underline{x})$. There seems not yet a name for this ring in literatures. For brevity, let us call $R(X, x)$ the ring of uniformization for $(X, \underline{x})$. We shall see that the ring of uniformization is finitely generated over $\mathbf{Z}$ and its transcendental degree (over $\mathbf{Q}$ ) is bounded. In fact, we shall find a universal ring $R_{g, n}$ such that the ring of uniformization $R(X, \underline{x})$ for any $(X, x) \in \mathfrak{M}_{g, n}$ is a homomorphic ımage of $R_{g, n}$. In this way, we embed the Teichmüller space into the affine variety $\operatorname{Hom}\left(R_{g, n}, \mathbf{R}\right)$.

(6.4) Before we can state the result, we need one more notion on spin structure on $X$. A spin on a Riemann surface $X$ is, by definition, a line bundle $\mathcal{L}$ on $X$ scuh that $\mathcal{L}^{2} \simeq$ canonical bundle of $X$. The group $\operatorname{Hom}\left(\pi_{1}(X), \mathbf{Z} / 2 \mathbf{Z}\right)(\simeq$ the 2-torsions of the Jacobi variety of $X)$ acts simple and transitively on the set of spins over $X$. Replacing $(X, \underline{x})$ by a spin Riemann surface $(X, \underline{x}, \mathcal{L})$, we can reformulate the definitions of $\mathfrak{M}_{g, n}$ and $\mathcal{T}_{g, n}$ and obtain the moduli space $\widetilde{\mathfrak{M}}_{g, n}$ and the Teichmüller space $\tilde{\mathcal{T}}_{g, n}$ for spin Riemann surfaces, respectively. One can show that the spin group $\operatorname{Hom}\left(\Gamma_{g}, \mathbf{Z} / 2 \mathbf{Z}\right)$ acts freely on the set of components of $\widetilde{\mathfrak{M}}_{g, n}$ and $\tilde{\mathcal{T}}_{g, n}$ such that their quotient spaces are $\mathfrak{M}_{g, n}$, and $\mathcal{T}_{g, n}$ respectively. (Actually, this follows from the "liftability" of a group in $P S L_{2}(\mathbf{R})$ to $S L_{2}(\mathbf{R})$, discussed in the proof of the next main theorem.)

(6.5) We now state the main theorem of the present paper. For the given $g \geqq 0$ and $n \geqq 0$ with $2-2 g-n<0$, let us introduce a ring by

$$
R_{g, n}:=R\left(\Gamma_{g, n}, \mathrm{SL}_{2}\right) /\left(s\left(c_{1}\right)+2, \ldots, s\left(c_{n}\right)+2\right)
$$

where $R\left(\Gamma_{g, n}, \mathrm{SL}_{2}\right)$ is the universal character ring (3.1.1) for the group $\Gamma_{g, n}(6.2 .1)$. Since $R\left(\Gamma_{g, n}, \mathrm{SL}_{2}\right)$ is finitely generated over $\mathbf{Z}\left((3.3)\right.$ Prop.), so is the $R_{g, n}$. The spin group $\operatorname{Hom}\left(\Gamma_{g}, \mathbf{Z} / 2 \mathbf{Z}\right)$ (= the subgroup of $\operatorname{Hom}\left(\Gamma_{g, n}, \mathbf{Z} / 2 \mathbf{Z}\right)$ through the identification $\left.\Gamma_{g} \simeq \Gamma_{g, n} /\left\langle c_{i}\right\rangle\right)$ and the mapping class group (6.2.3) act naturally on the ring $R_{g, n}$ by letting:

$$
\begin{gathered}
(\chi, s(\gamma)) \in \operatorname{Hom}\left(\Gamma_{g}, \mathbf{Z} / 2 \mathbf{Z}\right) \times R_{g, n} \mapsto \chi(\gamma) s(\gamma) \in R_{g, n} \\
(\alpha, s(\gamma)) \in \operatorname{Out}^{+}\left(\Gamma_{g, n}\right) \times R_{g, n} \mapsto s\left(\alpha^{-1}(\gamma)\right) \in R_{g, n}
\end{gathered}
$$

THEOREM. The spın Teichmüller space $\tilde{\mathcal{T}}_{g, n}$ is real analytically isomorphic to a union of connected components (in classical topology) of the real affine variety Hom 
$\left(R_{g, n}, \mathbf{R}\right)$. The embedding map

$$
\iota: \tilde{\mathcal{T}}_{g, n} \subset \operatorname{Hom}\left(R_{g, n}, \mathbf{R}\right)
$$

is equivariant with the actions of the spin group $\operatorname{Hom}\left(\Gamma_{g}, \mathbf{Z} / 2 \mathbf{Z}\right)$ and of the mapping class group $\mathrm{Out}^{+}\left(\Gamma_{g, n}\right)$. Furtheremore, the ८ satisfies the properties

$$
R(X, \underline{x})=\iota(t)\left(R_{g, n}\right)
$$

for any point $t(=$ the class of $(X, \alpha, \underline{x}))$ in $\tilde{\mathcal{T}}_{g, n}$. That is: the ring of uniformization $R(X, \underline{x})$ of $(X, \underline{x})(6.3 .1)$ is equal to the homomorphıc image of $R_{g, n}$ at the point in $\tilde{\mathcal{T}}_{g, n}$ representing the class of $(X, \alpha, \underline{x}, \mathcal{L})$ for any isomorphism $\alpha: \Gamma_{g, n} \simeq \pi_{1}(X \backslash\{\underline{x}\})$ and any spin bundle $\mathcal{L}$ on $X$.

Corollary. For any $(X, \underline{x}) \in \mathfrak{M}_{g, n}$, the ring of uniformization $R(X, \underline{x})$ is finitely generated over $\mathbf{Z}$ and

$$
0 \leq \operatorname{tr} \cdot \operatorname{deg}_{\mathbf{Q}}(\mathbf{Q} \otimes \mathbf{z} R(X, \underline{x})) \leq 6 g-6+n .
$$

An outline of a proof of the theorem. We repeat the ideas of description the Teichmüller spaces, started by Fricke [F-K] and developed by many authors including Keen $[\mathrm{K}]$, Helling [H], Okumura [O], Komori [Ko] and Sepällä-Sorvalli [S-S].

In view of the uniformization theorem of Poincaré, the $\mathcal{T}_{g, n}$ can be described as $\left\{\begin{array}{l}\text { set of }(\Gamma, \bar{\rho}), \text { where } \Gamma \text { is a discrete subgroup of } \mathrm{PSL}_{2}(\mathbf{R}) \text { and } \bar{\rho} \text { is an isomorphism } \\ \bar{\rho}: \Gamma_{g, n} \simeq \Gamma \text { such that }\left|\operatorname{tr} \bar{\rho}\left(c_{i}\right)\right|=2, \text { modulo the equivalence }(\Gamma, \bar{\rho}) \sim\left(\Gamma^{\prime}, \bar{\rho}^{\prime}\right) \text { if } \\ \text { there is } g \in \mathrm{PGL}_{2}(\mathbf{R}) \text { such that } g^{-1} \Gamma g=\Gamma^{\prime} \text { and } g^{-1} \bar{\rho} g=\bar{\rho}^{\prime} .\end{array}\right\}$

In order to adapt to the invariant theory developped in $\$ 1-5$, we want to lift the $\bar{\rho}: \Gamma_{g, n} \rightarrow \mathrm{PSL}_{2}(\mathbf{R})$ to $\rho: \Gamma_{g, n} \rightarrow \mathrm{SL}_{2}(\mathbf{R})$. This is possible for any group $\Gamma$ without 2 -torsions $[\mathrm{Cu}]$ (cf. $[\mathrm{A}-\mathrm{A}-\mathrm{S}],[\mathrm{F}],[\mathrm{Kr} 2],[\mathrm{Pa}]$ ). The ambiguity of the lifting $\bar{\rho}$ to $\rho$ is obviously described by the action of $\chi \in \operatorname{Hom}\left(\Gamma_{g, n}, \mathbf{Z} / 2 \mathbf{Z}\right)$ on $\rho$ by letting $(\chi \rho)(\gamma)=\chi(\gamma) \rho(\gamma)$. On the other hand, it is shown by Okumura [O1,2] that the signs of $\operatorname{tr}\left(\rho\left(c_{i}\right)\right)$ can be chosen to be simultaneously negative: $\operatorname{tr}\left(\rho\left(c_{i}\right)\right)=-2$ for $1 \leq i \leq n$. Choosing only such liftings, the ambiguity is reduced to $\operatorname{Hom}\left(\Gamma_{g}, \mathbf{Z} / 2 \mathbf{Z}\right)$. On the other hand, the subgroup $\rho\left(\Gamma_{g, n}\right) \subset \mathrm{SL}_{2}(\mathbf{R})$ is non-abelian and irreducible. So in view of (4.2) Assertion, we see that the restriction of the map $\pi_{\Gamma_{g, n}}$ (4.1.2) on the set $\left\{\rho \in \operatorname{Hom}\left(\Gamma_{g, n}, \mathrm{SL}_{2}(\mathbf{R})\right) \mid\right.$ faithful, discrete and $\operatorname{tr} \rho\left(c_{i}\right)+2=0$ for $\left.1 \leq i \leq n\right\}$ induces a $\mathrm{PGL}_{2}(\mathbf{R})$-fibration over the base space of $\operatorname{Hom}\left(R\left(\Gamma_{g, n}, \mathrm{SL}_{2}\right), \mathbf{R}\right) \cap\left\{s\left(c_{1}\right)+2=\cdots=\right.$ $\left.s\left(c_{n}\right)+2=0\right\}\left(=\operatorname{Hom}\left(R_{g, n}, \mathbf{R}\right)\right)\left((5.5)\right.$ Theorem C). So the $\tilde{\mathcal{T}}_{g, n}\left(\right.$ as the $\mathrm{PGL}_{2}(\mathbf{R})=$ quotient set) is embedded into the real affine algebraic variety $\operatorname{Hom}\left(R_{g, n}, \mathbf{R}\right)$ by the map $\pi_{\Gamma_{g, n}}(\rho)=: \iota([\rho])((5.5 .1))$. The fact that the $\tilde{\mathcal{T}}_{g, n}$ is an open subset of $\operatorname{Hom}\left(R_{g, n}, \mathbf{R}\right)$ follows from the fact $\pi_{\Gamma}^{-1}\left(\tilde{\mathcal{T}}_{g, n}\right)$ in open in the representation variety ([W] in case of $n=0$, [M-S] in case of $n>0$ ) and by an application of Luna's transversal slice theorem ([Lu],[Sl]) to the fibration $\pi_{\Gamma}((5.5)$ Theorem C). Closedness follows from the Jörgensen's inequalities for discrete subgroups of $\mathrm{PSL}_{2}(\mathbf{R})$ in terms of traces $[\mathrm{J}]$ and the

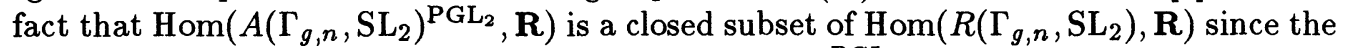
ring homomorphism $R\left(\Gamma_{g, n}, \mathrm{SL}_{2}\right) \otimes \mathbf{Q} \rightarrow A\left(\Gamma_{g, n}, \mathrm{SL}_{2}\right)^{\mathrm{PGL}_{2}} \otimes \mathbf{Q}$ is surjective. 
The equality (6.5.5) is an immediate consequence of the definitions of $\iota$ and of the ring of uniformization (6.3.1) (cf. (5.5) Theorem C). The corollary is a consequence of the fact $R_{g, n}$ is finitely generated and tr. $\operatorname{deg}_{\mathbf{Q}}\left(R_{g, n} \otimes \mathbf{Q}\right)=6 g-6+n$.

QUESTION. Does the property (6.5.5) characterize the embedding $\iota$ (6.5.4) up to the actions of $\operatorname{Hom}\left(\Gamma_{g}, \mathbf{Z} / 2 \mathbf{Z}\right)$ and $\mathrm{Out}^{+}\left(\Gamma_{g, n}\right)$ ?

(6.6) Remark. Here one should be carefull about the fact that the semialgebraic structure on $\tilde{\mathcal{T}}_{g, n}$ may not determine the ring $R_{g, n}$ yet, but it determines only the ring $R_{g, n} / I_{g, n}$ where

$$
I_{g, n}:=\bigcap_{t \in \mathcal{T}_{g, n}} \operatorname{ker}(\iota(t))
$$

It seems to be an interesting question to determine the ideal $I_{g, n}$. Can it be a zero ideal or nilradical, or, at least, a minimal prime?

(6.7) Example. Let $g=n=1$. Then the coordinate ring $R_{1,1}$ and $\tilde{\mathcal{T}}_{1,1}$ are expressed as follows.

$$
\begin{aligned}
& R_{1,1} \simeq \mathbf{Z}[x, y, z] /\left(x^{2}+y^{2}+z^{2}-x y z\right) . \\
& \operatorname{Hom}\left(R_{1,1}, \mathbf{R}\right)=\{0\} \amalg H_{2} \amalg H_{2} \amalg H_{2} \amalg H_{2}
\end{aligned}
$$

where $x=s\left(a_{1}\right), y=s\left(b_{1}\right)$ and $z=s\left(a_{1} b_{1}\right)$ and the component $H_{2}$ is the hyperbolic plane. One has $\iota: \tilde{\mathcal{T}}_{1,1}=4 \times H_{2}$. The $\operatorname{spin}$ group $(\mathrm{Z} / 2)^{2}=\left(\varepsilon_{1}, \varepsilon_{2}, \varepsilon_{3}\right) \mid \varepsilon_{1} \varepsilon_{2} \varepsilon_{3}=1$ acts transitively on the components by letting $(x, y, z) \mapsto\left(\varepsilon_{1} x, \varepsilon_{2} y, \varepsilon_{3} z\right)$. The point $(x, y, z) \in$ $\tilde{\mathcal{T}}_{1,1}$ corresponds to the $\mathrm{PGL}_{2}(\mathbf{R})$-orbit of a representation $\rho \in \operatorname{Hom}\left(\Gamma_{1,1}, \mathrm{SL}_{2}(\mathbf{R})\right)$ with $\rho(a)=\left[\begin{array}{ll}\lambda & 0 \\ 0 & \mu\end{array}\right]$ and $\rho(b)=\left[\begin{array}{ll}p & q \\ 1 & r\end{array}\right]$ where $\lambda, \mu$ are solutions of the equation $\lambda^{2}-x \lambda+1=$ $0,\left[\begin{array}{l}p \\ r\end{array}\right]=\frac{1}{\lambda-\mu}\left[\begin{array}{cc}\mu & -1 \\ -\lambda & 1\end{array}\right]\left[\begin{array}{l}y \\ z\end{array}\right], q=-\frac{\Delta}{\delta}$ and $\Delta=-4$. The point $\{0\}$ corresponds to a representation $\rho: \Gamma_{1,1} \rightarrow \mathrm{SO}(2)$ such that $\operatorname{tr} \rho(a)=\operatorname{tr} \rho(a b)=0$ and $\operatorname{tr} \rho\left(a b a^{-1} b^{-1}\right)=-2$. It may be clear that the homomorphism $\iota(t): R_{1,1} \rightarrow \mathbf{R}$ is injective for a generic $t$ in $\tilde{\mathcal{T}}_{1,1}$. Hence the property (6.5.5) determine the coordinate ring $R_{1,1}$ uniquely.

(6.8) We have now two algebraic structures on the moduli spaces: the classical one on $\mathfrak{M}_{g, n}$ coming from the field of definition of the curves, and the new one on $\mathcal{T}_{g, n}$ coming from the ring of uniformization of the curves. Symbolically saying, they are transcendentally different, since the group $\mathrm{Out}^{+}\left(\Gamma_{g, n}\right)$ is infinite. The attempt of the author, which motivated him to introduce the real algebraic coordinate system $R_{g, n}$ on $\mathcal{T}_{g, n}$ studied in the present paper, is to construct real automorphic forms on $\mathcal{T}_{g, n}$ with respect to the mapping class group (see [S2]). The original end is yet impenetrable. We restrict ourselves here only to pose some related problems.

The first one is the following.

Problem. Study the point $(X, \underline{x})$ of $\mathfrak{M}_{g, n}$ (and $\mathcal{T}_{g, n}$ ), which is algebraic in the both sense of $\mathfrak{M}_{g, n}$ and $\mathcal{T}_{g, n}$. (Here the $(X, \underline{x})$ is algebraic in the sense of $\mathcal{T}_{g, n} \underset{\text { def }}{\Longleftrightarrow}$ if tr. $\operatorname{deg}(R(X, \underline{x}))=0$.) Can the point be characterized in terms of certain symmetry of $(X, x)$, its Jacobian, or ..., etc.? Is there any process to list up such points? 
In case of $n=0$, there is an intermediate space between $\mathfrak{M}_{g}$ and $\mathcal{T}_{g}$. That is the period domain (inside Siegel upper half space) of abelian integrals. Does the algebraicity of a $(X, \underline{x})$ in the above two senses of $\mathfrak{M}_{g}$ and $\mathcal{T}_{g}$ imply also the algebraicy of the periods? Even stronger, any two algebraicities imply the third?

For instance, it is classical that in the case $g=1, n=0$, if the proportion $\omega_{1} / \omega_{2}$ of basic periods of an elliptic curve $E$ is imaginary quadratic if and only if $J(E)$ has a non trivial endomorphism, and then the absolute invariant $J(E) \in \mathfrak{M}_{1}$ is an imaginary quadratic number. Concerning those questions, one may be referred to the classical literatures on complex multiplications as well as [C-W] [Wf] [Shi] [T1,2] and their references. (6.9) To formulate the second problem, we introduce a completion of the universal character ring. For any normal subgroup $\Gamma^{\prime}$ of $\Gamma$, we define

$$
I\left(\Gamma^{\prime}, \Gamma\right):=\text { the ideal in } R(\Gamma) \text { generated by } s(\gamma \delta)-s(\delta) \text { for }{ }^{\forall} \gamma \in \Gamma^{\prime} \text { and }{ }^{\forall} \delta \in \Gamma \text {. }
$$

Let $\left\{\Gamma_{i}\right\}_{i \in \Lambda}$ be an inductive system (i.e. ${ }^{\forall} i, j \in \Lambda^{\exists} k \in \Lambda$ s.t. $\Gamma_{k} \subset \Gamma_{i} \cap \Gamma_{\jmath}$ ) of normal subgroups of $\Gamma$ and let $\widehat{\Gamma}:=\lim _{i \in \Lambda} \Gamma / \Gamma_{i}$. Then we define

$$
R(\widehat{\Gamma}):=\lim _{i \in \Lambda} R(\Gamma) / I\left(\Gamma_{\imath}\right)
$$

Problem. Study the variety $\operatorname{Spec}(R(\widehat{\Gamma}))$ and the action of $\operatorname{Out}(\widehat{\Gamma})$ on it.

Example. Let $\Gamma$ be the infinite cyclic group $\mathbf{Z}$. Then one has:

$$
R(\widehat{\mathbf{Z}}) \simeq \varliminf_{n}^{\lim } \mathbf{Z}[x] /\left(P_{n}(x)\right), \text { where } P_{n}(x):=\prod_{0<i \leq[n / 2]}(x-\cos (2 i \pi / n)) .
$$

Therefore $\operatorname{Hom}^{c}(R(\widehat{\mathbf{Z}}), \mathbf{R})=\bigcup_{n=1}^{\infty}\{\cos (2 i \pi / n) \mid 1 \leq i \leq[n / 2]\}$ is a dense subset of the compact interval $[-2,2]$. The Galois group $\operatorname{Gal}(\tilde{\mathbf{Q}} \mid \mathbf{Q})=\widehat{\mathbf{Z}}^{*}=\operatorname{Out}(\widehat{\mathbf{Z}})$ acts on the space.

\section{REFERENCES}

[A] W. Abikoff. The real analytic theory of Teichmüller space, Springer Lecture Notes Math., 820 (1980).

[A-A-S] W. Abikoff, Appel, K. and Schupp, P.: Lifting surface groups to SL $(2, C)$, Lecture Notes in Math., Springer 971 (1983).

[B-L] Hyman Bass and Alexander Lubotzky: Automorphism of groups and of schemes of finite type, Israel J. of Math., 44 no.1 (1983), 1-22.

[B] Hyman Bass: Group of integral representation type, Pacific J. Math. 86 (1980), 15-51.

[Be] Beardon, Alan F: The geometry of discrete groups., GTM, Springer Verlag, 1983.

[Br] G.W. Brumfiel: The real spectrum compactification of Teichmüller space, Contem. Math., 74 (1988), 51-75.

[C-W] Paula Cohen and Jürgen Wolfart: Modular embedding for some non-arithmetic Fuchsian groups, Preprint IHES/M/88/57, Nov. 1988.

[Cu] Marc Culler: Lifting representations to covering groups., Adv. Math., 59 (1986), 64-70.

[C-S] Marc Culler and Peter B. Shalen: Varieties of group representations and splittings of 3manifolds, Annals of Math., 117 (1983), 109-146.

[D] Stephen Donkin: Invariants of several matrices, Invent. Math. 110 (1992), 389-401. 
[F] G. Faltings: Real projective structures on Riemann surfaces, Compos. Math., 48 (1983), 223269.

[F] Formanec: The center of the ring of $4 \times 4$ generic matrices, J. Alg. 62 (1980), 304-319.

[F-K] Robert Fricke and Felix Klein: Vorlesungen uber die Theorie der Automorphen Funktionen, 1 pp.365-370, Leipzig: B.G. Teubner 1897. Reprint: New York: Johnson Reprint Corporation, Academic Press, 1965.

[G] William Goldman: Topological components of spaces of representations, Inventionen Math., 93 (1988), 557-607.

[G-M] F. Gonzáles-Acuña and José María Montesinos-Amilibia: On the character variety of group representations in $\operatorname{SL}(2, C)$ and $\operatorname{PSL}(2, C)$, Math. Z. 214 (1993), 627-652.

[G-Y] J.H. Grace and A. Young: The algebra of invariants, Cambridge Univ. Press, New York, 1903.

[H1] Heinz Helling: Diskrete Untergruppen von $\mathrm{SL}_{2}(\mathbf{R})$, Inventiones math., 17 (1972), 217-229.

[H2] Heinz Helling: Ueber den Raum der kompakten Riemannschen flächen vom Geschlecht 2, J. reine angew. Math., 268/269 (1974), 286-293.

[Ho1] Robert Horowitz: Characters of free groups represented in the two dimensional linear group, Comm. Pure Apll. Math. 25 (1972), 635-649.

[Ho2] Robert Horowitz: Induced automorphisms of Fricke characters of free groups, Trans. Am. Math. Soc., 208 (1975), 41-50.

[J] J. Jørgensen: On discrete groups of Möbius Transformations, Amer. J. Math., 98 (1976), 739749.

[K1] Linda Keen: Intrinsic Moduli, Ann. of Math., 84 (1966), 404-420.

[K2] Linda Keen: On Fricke Moduli, Advances in the theory of Riemann surfaces, Ann. of Math. Studies 66 (1971), 205-2024.

[K3] Linda Keen: A correction to "On Fricke Moduli", Proc. Amer. Math. Soc., 40 (1973), 60-62.

[K4] Linda Keen: A rough fundamental domain for Teichmüller spaces, Bull. Amer. Math. soc., 83 (1977), 1199-1226.

[K-S] Kodaira, K. and Spencer, D.C.: On deformation of complex analytic structures, Ann. of Math., 67 (1958), 328-466.

[Ko] Yohei Komori: Semialgebraic description of Teichmüller space, Thesis, RIMS, Kyoto Univ., Jan., 1994.

[Kr1] Irvin Kra: Automorphic Forms and Kleinean Groups, W.A. Benjamin Inc., 1972.

[Kr2] Irvin Kra: On lifting of Kleinian groups to $\mathrm{SL}(2, \mathrm{C})$ in differential geometry and complex analysis (Rauch H. E. Memorial Volume), Springer, Berlin, Heidelberg, New York, (1985), 181193.

[Kr3] Irvin Kra: Maskit coordinate, Holom. Funct. \& Moduli 2, Math. Sci. Research Inst. Publication 11, Springer, 1992.

[L-M] Alexander Lubotzky and Andy R. Magid: Varieties of representations of finitely generated groups, Memoirs of the AMS, 58 No. 336, (1985).

[Lu] Luna: Slices etale, Bull. Soc. Math. France, Memoire, 33 (1973), 81-105.

[Ma] William Magnus: Rings of Fricke characters and automorphism groups of free groups, Math. Z. 170 (1980), 91-103.

[Mu1] David Mumford: Geometric Invariant Theory, Springer-Verlag, Berlin, Heidelberg 1965, Library of Congress Catalog Card Number 65-16690.

[Mu2] David Mumford: Curves and their Jacobians, Ann Arbor, The University of Michigan Press, 1975.

[N] Louise Nyssen: PSEUDO-REPRESENTATIONS, preprint, 1994.

[O1] Yoshihide Okumura: Fricke moduli and Keen moduli for Fuchsian groups and a certain class of quasi-Fuchsian groups, Master Thesis, Shizuoka Univ., 1986.

[O2] Yoshihide Okumura: Global real analytic coordinates for Teichmüller spaces, Thesis, Kanazawa Univ., 1989. 
[O3] Yoshihide Okumura: On lifting problem of Kleinean group into SL(2, C), Summer Seminar on Function Theory, July 1992.

[O4] Yoshihide Okumura: On the global real analytic coordinates for Teichmüller spaces, J. Math. Soc. Japan, 42 (1990), 91-101.

[P-B-K] V.P. Platonov and V.V. Benyash-Krivets: Characters of representations of finitely generated groups, Proc. of Steklov Inst. of Math., (1991) 203-213.

[Pr1] Claudio Procesi: Finite dimensional representations of algebras, Israel J. of Math. 19 (1974), 169-182.

[Pr2] Claudio Procesi: Invariant theory of $n \times n$ matrices, Adv. Math. 19 (1976), 306-381.

[S1] Kyoji Saito: Moduli space for Fuchsian groups, algebraic analysis, II Academic Press (1988), $735-787$.

[S2] Kyoji Saito: The limit element in the configuration algebra for a discrete group, A précis: Proc. ICM90, Kyoto (1990), 931-942. Preprint: RIMS-726, Nov. 1990.

[S3] Kyoji Saito: The Teichmüller space and a certain modular function from a view point of group representations, Alg. Geom. and relate Topics, Proc. Int. Symp., Inchoen, Republic of Korea, 1992.

[S4] Kyoji Saito: Representation variety of a finitely generated group into $S L_{2}$ or $G L_{2}$, Preprint RIMS-958, 1993.

[S5] Kyoji Saito: Algebraic Representation of the Teichmüller Spaces, The Grothendieck Theory of Dessins d'Enfants, Edited by L. Schneps, London Math. Soc. Lec. Note Ser. 200, Cambridge Univ. Press, 1994.

[Sc] Paul Schmutz: Die Parametriserung den Teichmüllerschen Räumen.

[S-S1] Mika Seppälä and Tuomas Sorvali: Parametrization of Möbius groups acting in a disk, Comment. Math. Helvetici 61 (1986) 149-160.

[S-S2] Mika Seppälä and Tuomas Sorvali: Affine coordinates for Teichmüller spaces, Math. Ann. 284 (1989) 169-176.

[S-S3] Mika Seppälä and Tuomas Sorvali: Trace commutators of Möbius transformations, Math. Scand. 68 (1991), 53-58.

[S-S4] Mika Seppälä and Tuomas Sorvali: Geometry of Riemann surfaces and Teichmüller spaces, North-Holland and Mathematics Studies 169, 1992.

[Sl] Peter Slodowy: Der Scheibensatz für Algebraische transformationsgruppen, in DMV Seminar Band 13, Algebraic Transformation Groups and Invariant Theory, edited by H. Kraft, P. Slodowy and T.A. Springer, Birkhäuse (1989), 89-113.

[T1] Kisao Takeuchi: On some discrete subgroups of $\mathrm{SL}_{2}(\mathbf{R})$, J. Fac. Sci. Univ. Tokyo, Sec. I, 16 (1969), 97-100.

[T2] Kisao Takeuchi: Fuchsian groups contained in $\mathrm{SL}_{2}(\mathbf{Q})$, J. Math. Soc. Japan, Vol. 23, No. 1 (1971), 82-94.

[V] H. Vogt: Sur les invariants, fondamentaux des équations différentielles linéaires du second ordre, Ann. Sci. Ecole Norm. Sup. (3)6, Suppl. 3-72 (1889) (Thèse, Paris).

[W] Andre Weil: On discrete subgroups of Lie groups. I. Ann. Math. 72 (1960), 369-384, II. Ann. Math. 75 (1962) 578-602.

[Wf] Jürgen Wolfart: Eine arithmetische Eigenschaft automorpher Formen zu gewiisen nichtarithmetischen Gruppen, Math. Ann. 62 (1983), 1-21.

[Wo1] Scott Wolpert: The Fenchel-Nielsen deformation, Ann. of Math. 115 (1982), 501-528.

[Wo2] Scott Wolpert: On the symplectic geometry of deformation of a hyperbolic surface, Ann. of Math., 117 (1983), 207-234.

[Wo3] Scott Wolpert: Geodesic length functions and the Nielsen problem, J. Diff. Geom. 25 (1987), 275-296.

[Z-V-C] Ziechang, Voght and Colderway: Lecture Note Math., Springer, 122, 835 \& 875. 
RIMS, KYOTO UNIVERSITY KITASHIRAKAWA, SAKYO-KU КYОTO 606 